\title{
Elasticity of randomly diluted honeycomb and diamond lattices with bending forces
}

\author{
Danilo B. Liarte \\ Laboratory of Atomic and Solid State Physics, Cornell University, Ithaca, NY, \\ USA \\ Institute of Physics, University of São Paulo, São Paulo, SP, Brazil
}

\section{O. Stenull}

Department of Physics and Astronomy, University of Pennsylvania, Philadelphia, PA, USA

\section{Xiaoming Mao}

Department of Physics, University of Michigan, Ann Arbor, MI, USA

\section{T. C. Lubensky}

Department of Physics and Astronomy, University of Pennsylvania, Philadelphia, PA, USA

January 2016

\begin{abstract}
We use numerical simulations and an effective-medium theory to study the rigidity percolation transition of the honeycomb and diamond lattices when weak bond-bending forces are included. We use a rotationally invariant bond-bending potential, which, in contrast to the Keating potential, does not involve any stretching. As a result, the bulk modulus does not depend on the bending stiffness $\kappa$. We obtain scaling functions for the behavior of some elastic moduli in the limits of small $\Delta \mathcal{P}=1-\mathcal{P}$, and small $\delta \mathcal{P}=\mathcal{P}-\mathcal{P}_{c}$, where $\mathcal{P}$ is an occupation probability of each bond, and $\mathcal{P}_{c}$ is the critical probability at which rigidity percolation occurs. We find good quantitative agreement between effective-medium theory and simulations for both lattices for $\mathcal{P}$ close to one.
\end{abstract}

Submitted to: J. Phys.: Condens. Matter 


\section{Introduction}

Concepts associated with the rigidity percolation transition of random elastic networks [1, 2] have been applied in many branches of science, such as amorphous solids 3, 4, granular materials [5, 6, mineralogy [7, networks of semi-flexible polymers [8, 9] and the mechanics of living cells [10, 11, 12]. The archetype of this transition [13, 14, 15, 9, 16, 17, occurs in periodic lattices in which bonds consisting of centralforce springs are populated with probability $\mathcal{P}$. For $\mathcal{P}$ below a threshold $\mathcal{P}_{c}$, the lattice loses rigidity, and some or all of its elastic moduli vanish because floppy regions prevent rigid ones from percolating.

The homogeneous honeycomb and diamond lattices (Fig. 1) with only nearest-neighbor bonds are strongly under-coordinated. The average coordination number $z$ of both the honeycomb and diamond lattices is less than the Maxwell limit [18, 19, $z_{c}=2 d$ for central forces, where $d$ is the spatial dimension, below which lattices under periodic boundary conditions develop zero-frequency "floppy" modes. The honeycomb lattice with 2 sites per unit cell and $z=3$ has a deficiency of one bond, and the diamond lattice with 2 sites per unit cell and $z=4$ has a deficiency of two bonds per unit cell relative to the Maxwell limit. As a result, they have an extensive number of zero modes and no resistance to shear distortions; but curiously because of the special geometry of their lattices, they both have a non-zero bulk modulus $B$. Thus extra forces, such as next-nearest-neighbor central forces or bending forces favoring a particular angle between pairs of bonds sharing common endpoints, are required for mechanical stability. Here, using both effective medium theory (EMT) and numerical simulations, we study the properties of randomly bond-diluted central-force honeycomb and diamond lattices with added bending forces characterized by a local bending stiffness $\kappa$, focussing in particular on behavior near zero dilution $(\mathcal{P} \approx 1)$ and near the rigidity threshold at $\mathcal{P}=\mathcal{P}_{c}$. We find that the bulk modulus of both lattices can be expressed near $\mathcal{P}=1$ as a scaling function of $\kappa /(1-\mathcal{P})^{n}$ with $n=1$, much like the shear modulus in the diluted kagome lattice with bending forces [16 where $n=2$ rather than 1. The shear moduli of the honeycomb and diamond lattices, on the other hand exhibit, no simple scaling form and approach zero even at $\mathcal{P}=1$ as $\kappa \rightarrow 0$ as required. We find that the bulk moduli, much like the shear moduli in the Mikado model 20, 21, 22 and the diluted triangular 9 and kagome lattices with bending [16], exhibit crossover from stretching dominated affine response to bending dominated nonaffine response with decreasing $\mathcal{P}$, reaching a maximum nonaffinity at the rigidity threshold. The shear modulus, which vanishes with $\kappa$, on the other hand, always exhibits bending and thus non-affine response.
Bending forces effectively couple next-nearest neighbor sites, thereby increasing the effective $z$ to values above the central-force critical value, $z_{c}$, providing elastic stability, and eliminating all but the trivial zero modes of rigid translation and rotation. In contrast to Keating potentials [23], bending forces do not depend on the length of bonds and, therefore, do not contribute to the bulk modulus at $\mathcal{P}=1$. The Keating potential is rotational invariant. Bending forces are also, as we show in the Appendix.

(a)

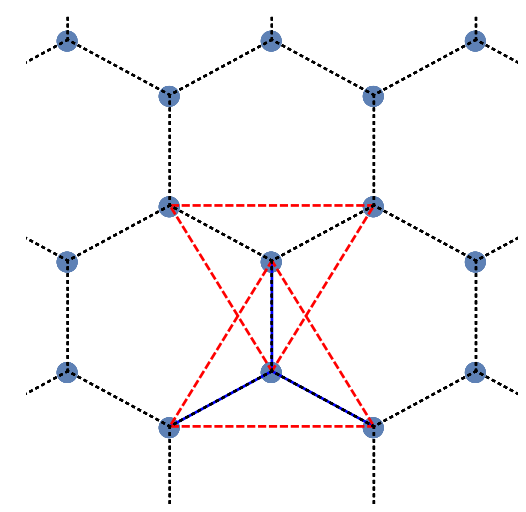

(b)

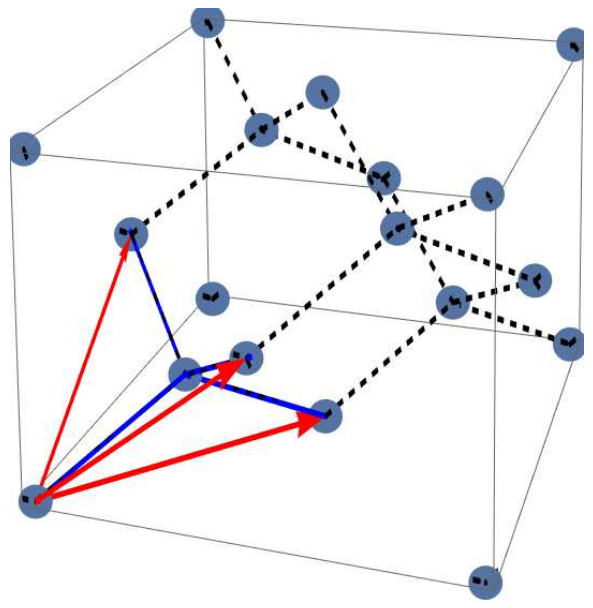

Figure 1. a) Crystal structure of the honeycomb net. b) Conventional cubic cell of the diamond lattice.

\section{Model}

The two-dimensional honeycomb lattice (Fig. 14) is a triangular Bravais lattice with a two-point basis [24]. It is defined by a set of primitive vectors, e.g. $\boldsymbol{a}_{1}=\sqrt{3}(1,0)$, and $\boldsymbol{a}_{2}=(\sqrt{3} / 2)(1, \sqrt{3})$, along with the positions of the atoms within each primitive cell, $\boldsymbol{c}_{1}=(0,0)$, and $\boldsymbol{c}_{2}=(0,1)$. The length of each bond in the lattice is $\ell_{0}=1$. The three-dimensional diamond lattice can be represented as a face-centered cubic lattice with a two-point basis (Fig. 10). It can 
be defined by the primitive vectors $\boldsymbol{a}_{1}=(1 / 2)(0,1,1)$, $\boldsymbol{a}_{2}=(1 / 2)(1,0,1), \boldsymbol{a}_{3}=(1 / 2)(1,1,0)$, along with the two-point basis vectors $\boldsymbol{c}_{1}=(0,0,0)$, and $\boldsymbol{c}_{2}=$ $(1 / 4)(1,1,1)$. Bonds connecting nearest-neighbor sites are of length to $\ell_{0}=\sqrt{3} / 4$.

We consider the following lattice energy of interaction:

$E=E_{\text {stretch }}+E_{\text {bend }}$.

The first term is a sum of central-force interactions between nearest-neighbor pairs of sites $i$ and $j$, which in the harmonic limit are given by:

$E_{\text {stretch }}^{i j}=\frac{1}{2} k\left[\left(\boldsymbol{u}_{j}-\boldsymbol{u}_{i}\right) \cdot \hat{\boldsymbol{r}}_{i j}\right]^{2}$,

where $\hat{\boldsymbol{r}}_{i j}$ is the unit vector connecting sites $i$ and $j$ in the undeformed lattice, and $\boldsymbol{u}_{i}$ is a displacement vector. Each unit cell in the honeycomb lattice has three independent bonds (e.g., the blue lines of Fig. 1a), and in the diamond lattice, four independent bonds. The second term in Eq. (11) is a sum of bending energy interactions associated with two bonds, terminating on a common site $l$ and connecting nearestneighbor pairs of sites $(l, m)$, and $(l, k)$ :

$E_{\text {bend }}^{k l m} \equiv \frac{\kappa}{2}\left(\sin \beta_{0} \Delta \beta_{k l m}\right)^{2}$,

where $\beta_{0}$ is the equilibrium angle between bonds, and $\Delta \beta_{k l m}$ represents the difference between the angle between the bonds $(l, m)$ and $(l, k)$ and $\beta_{0}$. There are six such terms per primitive cell for the honeycomb lattice, and twelve for the diamond lattice. The bending interactions effectively couple next-nearestneighbors sites, as illustrated by the red dashed lines of Fig. 17. The factor $\sin \beta_{0}$ is a matter of convenience. It is equal to $\sqrt{3} / 2$ for the honeycomb lattice, and $2 \sqrt{2} / 3$ for the diamond lattice. Equation (3) may be written as a combination of displacement vectors, up to second order in $\boldsymbol{u}$, as

$$
\begin{aligned}
E_{\text {bend }}^{k l m}=\frac{\kappa}{2 \ell_{0}^{2}}\left\{\boldsymbol{u}_{l m} \cdot\left[\hat{\boldsymbol{r}}_{l k}-\left(\hat{\boldsymbol{r}}_{l k} \cdot \hat{\boldsymbol{r}}_{l m}\right) \hat{\boldsymbol{r}}_{l m}\right]\right. \\
\left.+\boldsymbol{u}_{l k} \cdot\left[\hat{\boldsymbol{r}}_{l m}-\left(\hat{\boldsymbol{r}}_{l k} \cdot \hat{\boldsymbol{r}}_{l m}\right) \hat{\boldsymbol{r}}_{l k}\right]\right\}^{2},
\end{aligned}
$$

where $\boldsymbol{u}_{l k}=\boldsymbol{u}_{k}-\boldsymbol{u}_{l}$.

The honeycomb lattice has four phonon branches, corresponding to the four degrees of freedom within each unit cell. In the limit $\kappa \rightarrow 0$, one of the two acoustic branches is floppy with zero frequency for all wavenumbers $\boldsymbol{q}=\left(q_{x}, q_{y}\right)$ in the Brillouin Zone. Figure $2 a$ shows a density plot of one of the acoustic branch frequencies for homogeneous $k=1$, and for $\kappa=\tilde{\kappa}=0.01$. Hereafter $k=1$ is assumed unless otherwise noted. Figure $2 \mathrm{~b}$ displays dispersion curves along symmetry lines $\Gamma M, \Gamma K$, and $K M, \kappa=0$ (dashed curves), and $\kappa=0.1$ (solid curves). Figure 3 show a $3 d$ plot of the two acoustic branches as a function of $q_{x}$ and $q_{y}$, for $\kappa=0.01$. The diamond (a)

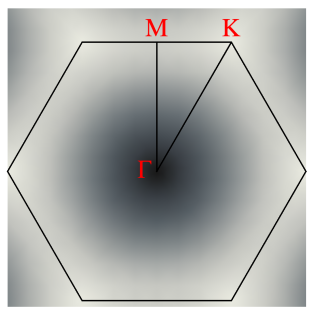

(b)

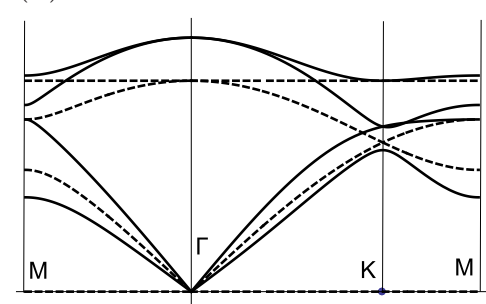

Figure 2. a) Density plot in the $q_{x} \times q_{y}$ plane of one of the honeycomb's acoustic modes for $k=1$, and $\kappa=0.01$. b) Dispersion curves along some symmetry lines for $k=1, \kappa=0$ (dashed curves), and $\kappa=0.1$ (solid curves).

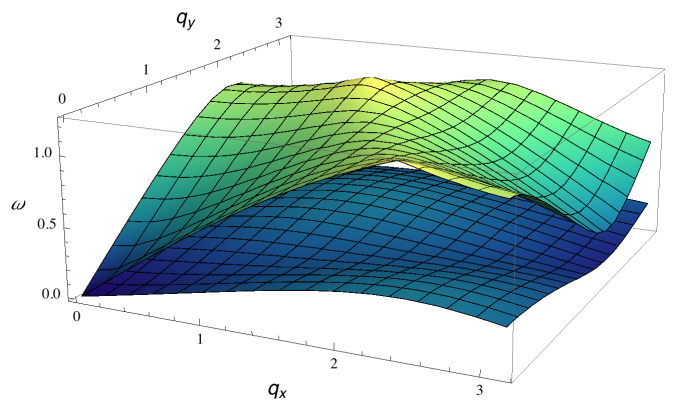

Figure 3. Three-dimensional plot of the acoustic branch frequencies of the honeycomb lattice for $k=1$ and $\kappa=0.01$.

lattice has six phonon branches, of which two are floppy when $\kappa \rightarrow 0$. Figure 4a shows diamond-lattice dispersion curves for $\kappa=0.01$, along symmetry lines $\Gamma X$ and $\Gamma L$. A sketch of the first Brillouin zone of the diamond lattice with five high symmetry points is displayed in Figure 4b. Notice that two largest and the two smallest eigenvalues are degenerate in both lines. These degeneracies can be broken along other less symmetrical lines.
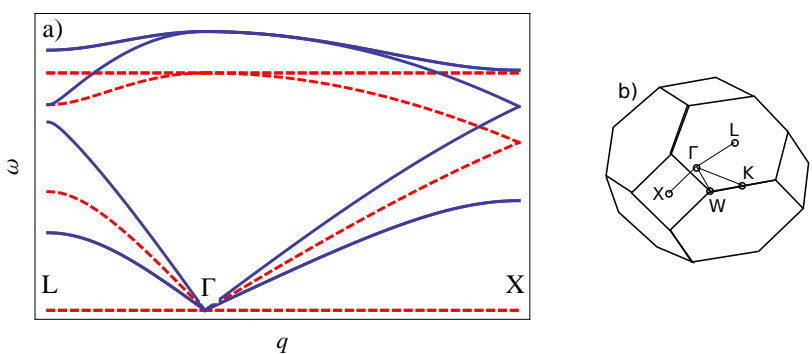

Figure 4. a) Dispersion curves of the diamond lattice along symmetry lines $L-\Gamma$ and $\Gamma-X$ The dashed lines represent $\kappa=0$ and the full lines $\kappa=0.01$. b) Sketch of the first Brillouin zone of the diamond lattice and high symmetry points $\Gamma, L, X$, $K$, and $W$. 


\section{Effective-medium theory and simulations}

We use an adaptation of the EMT developed in Ref. 25, 10, in which the spring constants of individual bonds and the bending constants of individual bond pairs are treated as independent random variables $\mathrm{t}$. The probability that a given bond is occupied is $\mathcal{P}$, and the probability distribution for the spring constant for any bond is

$P_{\text {spr }}\left(k^{\prime}\right)=\mathcal{P} \delta\left(k^{\prime}-k\right)+(1-\mathcal{P}) \delta\left(k^{\prime}\right)$.

Both bonds of a bond pair must be occupied in order for there to be a bending energy associated with the pair. Following the approximation of Refs. 25, 10, we set the probability that a given bond pair exists equal to $\mathcal{P}^{2}$. The probability distribution for the bending constant of an individual bond pair is

$P_{\text {bend }}=\mathcal{P}^{2} \delta\left(\kappa^{\prime}-\kappa\right)+\left(1-\mathcal{P}^{2}\right) \delta\left(\kappa^{\prime}\right)$.

The joint probability for both $k^{\prime}$ and $\kappa^{\prime}$ is then $P\left(k^{\prime}, \kappa^{\prime}\right)=P_{\text {spr }}\left(k^{\prime}\right) P_{\text {bend }}\left(\kappa^{\prime}\right)$.

In this EMT theory, each occupied bond and each occupied bond pair constitutes a constraint. Thus if each site has $z$ neighbors, there are $(z \mathcal{P} / 2) N$ bond constraints and $\left(z(z-1) \mathcal{P}^{2} / 2\right) N$ bond-pair constraints in a diluted lattices of $N$ sites. Since each site has $d$ translational degrees of freedom, the Maxwell count of the number of zero modes per site is

$f=d-\frac{z \mathcal{P}}{2}-\frac{z(z-1)}{2} \mathcal{P}^{2},$.

The EMT rigidity threshold is obtained by setting $f=0$ to produce

$\mathcal{P}_{c}=\frac{1}{2(z-1)}\left[\sqrt{1+\frac{8 d(z-1)}{z}}-1\right]$.

Thus, in the honeycomb lattice $\mathcal{P}_{c} \approx 0.60$ and the average coordination number at threshold, $z_{c}=z \mathcal{P}$, is 1.8; in the diamond lattice, $\mathcal{P}_{c} \approx 0.56$ and $z_{c} \approx 2.24$.

The EMT threshold should be compared with two other estimates of $z_{c}$ based on the Maxwell zero-mode count. Phillips [27, 28], who considered general off lattice networks, also treated bond pairs as independent and obtained an equation identical to Eq. (77) but without the $\mathcal{P}$ factors and with $z \rightarrow r$ interpreted as the average coordination number, $r=$ $\mathcal{P} z$. His estimate leads to $r_{c}=\sqrt{2 d}$ or $r_{c}=2\left(\mathcal{P}_{c}=\right.$ $0.67)$ and $r_{c}=2.45(\mathcal{P}=0.61)$ for the honeycomb and diamond lattices, respectively. Thorpe later showed [1] that treating each bond pair independently overcounts the number of bending constraints. In his

$\ddagger$ This version of CPA is widely used and accepted in treatments of rigidity percolation. We divide the interactions up into equivalency classes and average, so our effective Hamiltonian is writable as a sum of homomorphic parts. This version of CPA is equivalent to what Yonezawa and Ogadaki call the HCPA [26], which yields an analytical physical solution. analysis, a single pair of bonds, sharing a common site, contributes one constraint due to bending forces. Each additional bond sharing that site is constrained to have a particular orientation, and thus adds a total of $d-1$ constraints, which is the number of angles needed to specify a unit vector in $d$-dimensions. Therefore, the total number of bending-force constraints (for $r \geq 3$ ) reads,

$n_{\mathrm{B}}^{\mathrm{Th}}(r+1)=d-1+n_{\mathrm{B}}^{\mathrm{Th}}(r) \Rightarrow$

$n_{\mathrm{B}}^{\mathrm{Th}}(r)=(d-1) r-(2 d-3)$,

with

$r=\left(\sum_{i=2}^{z} i n_{i}\right) /\left(\sum_{i=2}^{z} n_{i}\right)$,

where $n_{i}$ is the number of $i$-coordinated sites. Note that in applying Eq. (10), one has to ensure that there are neither isolated nor 1-coordinated sites. Thorpe's counting, including $r / 2$ stretching constraints, gives $r_{c}=6(d-1) /(2 d-1)=2$ and $12 / 5=2.4$ for the honeycomb and diamond lattices respectively.

In effective-medium theory, the diluted lattice is modeled by a homogeneous lattice with stiffness constants $k_{m}$ and $\kappa_{m}$ satisfying a set of self-consistent equations that depend on a probability distribution $P\left(k^{\prime}, \kappa^{\prime}\right)$. We use an adaptation of the effectivemedium theories proposed in Ref. [13] to derive the set of effective-medium theory equations for $k_{m}$ and $\kappa_{m}$,25, 10, 16]:

$\frac{k_{m}}{k}=\frac{\mathcal{P}-a^{*}}{1-a^{*}}$,

$\frac{\kappa_{m}}{\kappa}=\frac{\mathcal{P}^{2}-b^{*}}{1-b^{*}}$,

where

$a^{*}=\frac{1}{z} \int_{1 B Z} \frac{d \boldsymbol{q}}{\tilde{v}_{0}} \operatorname{Tr}\left(\boldsymbol{D}_{s, \boldsymbol{q}} \cdot \boldsymbol{D}_{\boldsymbol{q}}^{-1}\right)$,

$b^{*}=\frac{1}{(z-1) z} \int_{1 B Z} \frac{d \boldsymbol{q}}{\tilde{v}_{0}} \operatorname{Tr}\left(\boldsymbol{D}_{b, \boldsymbol{q}} \cdot \boldsymbol{D}_{\boldsymbol{q}}^{-1}\right)$,

where $\tilde{v}_{0}$ is the volume of the first Brillouin zone. In Eqs. (14) and (15), $\boldsymbol{D}_{\boldsymbol{q}}$ is the translational invariant dynamical matrix in Fourier space:

$\boldsymbol{D}_{\boldsymbol{q}, \boldsymbol{q}^{\prime}}=N \delta_{\boldsymbol{q}, \boldsymbol{q}^{\prime}} \boldsymbol{D}_{\boldsymbol{q}}$,

$\boldsymbol{D}_{\boldsymbol{q}}=\boldsymbol{D}_{s, \boldsymbol{q}}+\boldsymbol{D}_{b, \boldsymbol{q}}$,

where $\boldsymbol{D}_{s, \boldsymbol{q}}$, and $\boldsymbol{D}_{b, \boldsymbol{q}}$ are contributions from the stretching and bending interactions respectively. They may be written as

$\boldsymbol{D}_{s, \boldsymbol{q}}=k_{m} \sum_{n=1}^{z} \boldsymbol{B}_{n, \boldsymbol{q}}^{s} \boldsymbol{B}_{n,-\boldsymbol{q}}^{s}$,

where,

$\boldsymbol{B}_{n, \boldsymbol{q}}^{s}=\left\{e^{-i \boldsymbol{q} \cdot \boldsymbol{f}_{n}} \boldsymbol{e}_{n},-\boldsymbol{e}_{n}\right\}$, 
and,

$$
\begin{gathered}
\boldsymbol{D}_{b, \boldsymbol{q}}=\frac{\kappa_{m}}{\ell_{0}^{2}} \sum_{1 \leq m<n \leq z}\left(\boldsymbol{B}_{m n, \boldsymbol{q}}^{b(i)} \boldsymbol{B}_{m n,-\boldsymbol{q}}^{b(i)}\right. \\
\left.+\boldsymbol{B}_{m n, \boldsymbol{q}}^{b(i i)} \boldsymbol{B}_{m n,-\boldsymbol{q}}^{b(i i)}\right)
\end{gathered}
$$

where $\ell_{0}$ is the lattice spacing, and

$$
\begin{aligned}
& \boldsymbol{B}_{m n, \boldsymbol{q}}^{b(i)}=\left\{e^{-i \boldsymbol{q} \cdot \boldsymbol{f}_{m}} \boldsymbol{e}_{n m}^{\perp}+e^{-i \boldsymbol{q} \cdot \boldsymbol{f}_{n}} \boldsymbol{e}_{m n}^{\perp},\right. \\
& \left.-\left(\boldsymbol{e}_{m n}^{\perp}+\boldsymbol{e}_{n m}^{\perp}\right)\right\}, \\
& \boldsymbol{B}_{m n, \boldsymbol{q}}^{b(i i)}=\left\{-\left(\boldsymbol{e}_{m n}^{\perp}+\boldsymbol{e}_{n m}^{\perp}\right), e^{i \boldsymbol{q} \cdot \boldsymbol{f}_{m}} \boldsymbol{e}_{n m}^{\perp}\right. \\
& \left.+e^{i \boldsymbol{q} \cdot \boldsymbol{f}_{n}} \boldsymbol{e}_{m n}^{\perp}\right\} .
\end{aligned}
$$

The vectors $\boldsymbol{e}_{n}$ and $\boldsymbol{f}_{m}$ connect nearest-neighbor sites and cells, respectively, \&, and

$\boldsymbol{e}_{m n}^{\perp}=\boldsymbol{e}_{m}-\left(\boldsymbol{e}_{m} \cdot \boldsymbol{e}_{n}\right) \boldsymbol{e}_{n}$.

These definitions imply that $a^{*}$ and $b^{*}$ are functions of the dimensionless ratio:

$\tilde{\kappa}_{m} \equiv \frac{\kappa_{m}}{k_{m} \ell_{0}^{2}}$,

rather than of $\kappa_{m}$ and $k_{m}$ separately. Equations (14), (15), and (17) lead to the following important relation between $a^{*}$ and $b^{*}$ :

$a^{*}+(z-1) b^{*}=(2 d) / z$.

At the rigidity threshold, $k_{m}=0$ and $\kappa_{m}=0$. Equations (12) and (13) then require $a^{*}=\mathcal{P}$ and $b^{*}=\mathcal{P}^{2}$, and Eq. (25) reduces to Eq, (7) at $f=0$ and yields Eq. (8) for $\mathcal{P}_{c}$.

\subsection{Simulations}

In the numerical portion of our work, we generate diluted honeycomb and diamond lattices on a computer. The systems sizes that we simulate range up to $100^{2}$ unit cells for the honeycomb lattice and $20^{3}$ unit cells for the diamond lattice. In all our simulations, periodic boundary conditions are applied. To facilitate the computations, we split up the elastic displacement $\boldsymbol{u}_{i}$ into an affine and a non-affine part,

$\boldsymbol{u}_{i}=\eta \boldsymbol{x}_{i}+\delta \boldsymbol{u}_{i}$,

where $\boldsymbol{x}_{i}$ is the equilibrium position of site $i$ in the absence of any applied deformation, $\eta$ is the deformation gradient tensor, and $\delta \boldsymbol{u}_{i}$ is the non-affine displacement. We fix the non-affine displacement of an arbitrarily chosen lattice site to be zero so that spurious zero modes associated with rigid translations of the lattice are suppressed. We apply shear and bulk deformations by choosing $\eta$ accordingly. For example, to apply shear to the honeycomb lattice, we chose the 2 diagonal components of $\eta$ to be zero and its

$\S$ We have chosen $\boldsymbol{e}_{1}=-\boldsymbol{c}_{2}, \boldsymbol{e}_{2}=\boldsymbol{a}_{2}-\boldsymbol{c}_{2}, \boldsymbol{e}_{3}=\boldsymbol{a}_{2}-\boldsymbol{a}_{1}-\boldsymbol{c}_{2}$, $f_{1}=c_{1}, f_{2}=a_{2}$, and $f_{3}=a_{2}-a_{1}$ for the honeycomb lattice, and $e_{1}=a_{1}-c_{2}, e_{2}=a_{2}-c_{2}, e_{3}=a_{3}-c_{2}, e_{4}=-c_{2}$, $f_{1}=a_{1}, f_{2}=a_{2}, f_{3}=a_{3}, f_{4}=c_{1}$ for the diamond lattice.
2 off-diagonal elements to be equal to $\gamma$, where $\gamma$ is the magnitude of the deformation which we set to $\gamma=0.01$. Then we relax the $\delta \boldsymbol{u}_{i}$ using a standard conjugate gradient algorithm that provides us with the equilibrium non-affine displacements $\delta \boldsymbol{u}_{i}^{\text {na }}$ in the presence of applied deformation. Feeding these back into the elastic model energy density (1), we obtain the shear and bulk moduli as function of $\mathcal{P}$ and $\kappa$. In addition to the elastic moduli, we also compute the so-called non-affinity parameter $\Gamma$ which measures the degree of non-affinity in the system under the applied deformation,

$\Gamma=\frac{1}{N \gamma^{2}} \sum_{i}\left(\delta \boldsymbol{u}_{i}^{\mathrm{na}}\right)^{2}$,

where $N$ is the total number of sites. Our numerical results will be displayed and discussed together with our EMT results as we move along.

\section{Results}

In our EMT, the bulk and shear moduli of the honeycomb and diamond lattices have the same form as functions of the effective medium spring and bending constants $k_{m}$ and $\kappa_{m}$ :

$$
\begin{aligned}
B & =A_{B} k_{m}=\frac{1}{3}\left(C_{11}+2 C_{12}\right), \\
\mu & =A_{\mu} \frac{k_{m} \kappa_{m} / \ell_{0}^{2}}{\beta k_{m}+\gamma \kappa_{m} / \ell_{0}^{2}} \\
& =\frac{A_{\mu}}{\beta} k_{m} \tilde{\kappa}_{m}\left(1+(\gamma / \beta) \tilde{\kappa}_{m}\right)^{-1}=C_{44} \\
& = \begin{cases}\frac{A_{\mu}}{\beta} \frac{\kappa_{m}}{\ell_{0}^{2}}, & \text { if } \tilde{\kappa}_{m} \ll 1, \\
\frac{A_{\mu}}{\gamma} k_{m}, & \text { if } \tilde{\kappa}_{m} \gg 1,\end{cases}
\end{aligned}
$$

where $C_{i j}$ are the standard Voigt elastic constants for a cubic crystal, $\tilde{\kappa}_{m}=\kappa_{m} /\left(k_{m} \ell_{0}^{2}\right), A_{B} \equiv A_{B, \mathrm{H}}=3 / 4$, $A_{\mu} \equiv A_{\mu \mathrm{H}}=27 / 2, \beta \equiv \beta_{\mathrm{H}}=2$, and $\gamma \equiv \gamma_{\mathrm{H}}=9$ for the honeycomb lattice, and $A_{B} \equiv A_{B, \mathrm{D}}=1 / 12$, $A_{\mu} \equiv A_{\mu, \mathrm{D}}=144, \beta \equiv \beta_{\mathrm{D}}=27$, and $\gamma \equiv \gamma_{\mathrm{D}}=192$ for the diamond lattice. Thus $B$ and $\mu$ are determined as a function of $\kappa$ and $\mathcal{P}$ once the EMT equations (12) and (13) are solved.

Figure 5 shows plots of numerical solutions of the EMT equations (solid lines) and simulations (symbols) for the bulk (left) and shear (right) moduli of the honeycomb lattice, as a function of the probability $\mathcal{P}$, for $\kappa=1,10^{-2}, 10^{-4}$, and $10^{-6}$ (in blue, red, yellow, and green respectively). We will keep the same color definitions in all subsequent plots. Figure [6 shows similar plots for the diamond lattice. In both cases, simulations and the EMT results agree well near $\mathcal{P}=1$. In the vicinity of the rigidity threshold $\mathcal{P}=\mathcal{P}_{c}$, the simulations display a decay that is different from that 

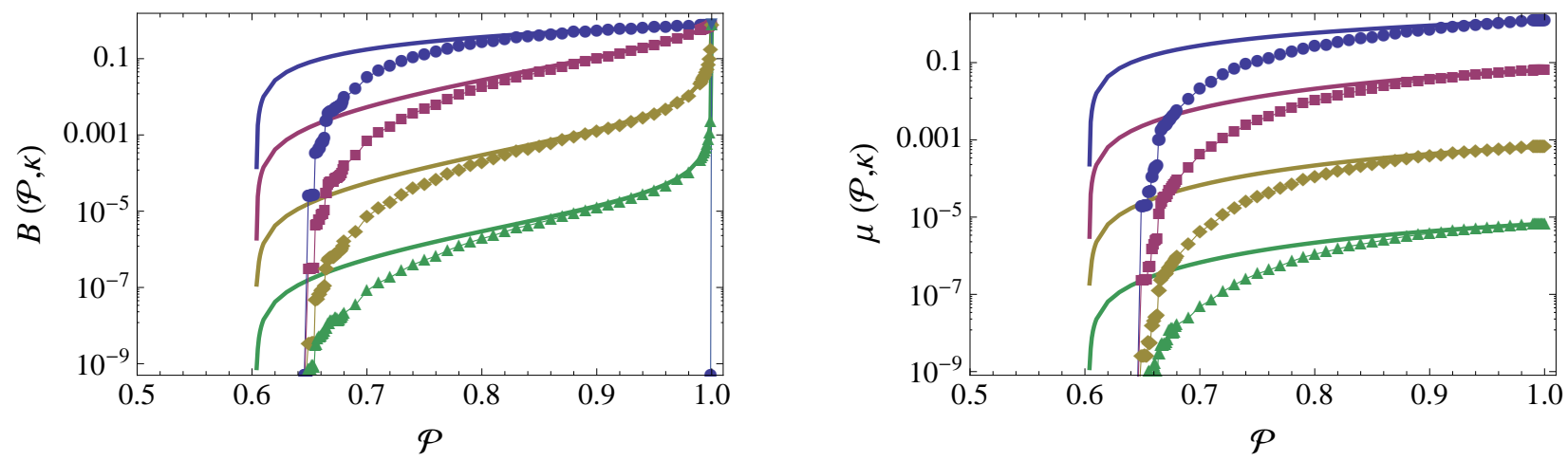

Figure 5. Bulk (on the left) and shear (on the right) moduli of the diluted honeycomb lattice as a function of $\mathcal{P}$, for $k=1$, and $\kappa=1$ (blue circles), $10^{-2}$ (red squares), $10^{-4}$ (yellow diamonds), and $10^{-6}$ (green triangles), from both simulations (symbols), and effective-medium theory (solid lines).
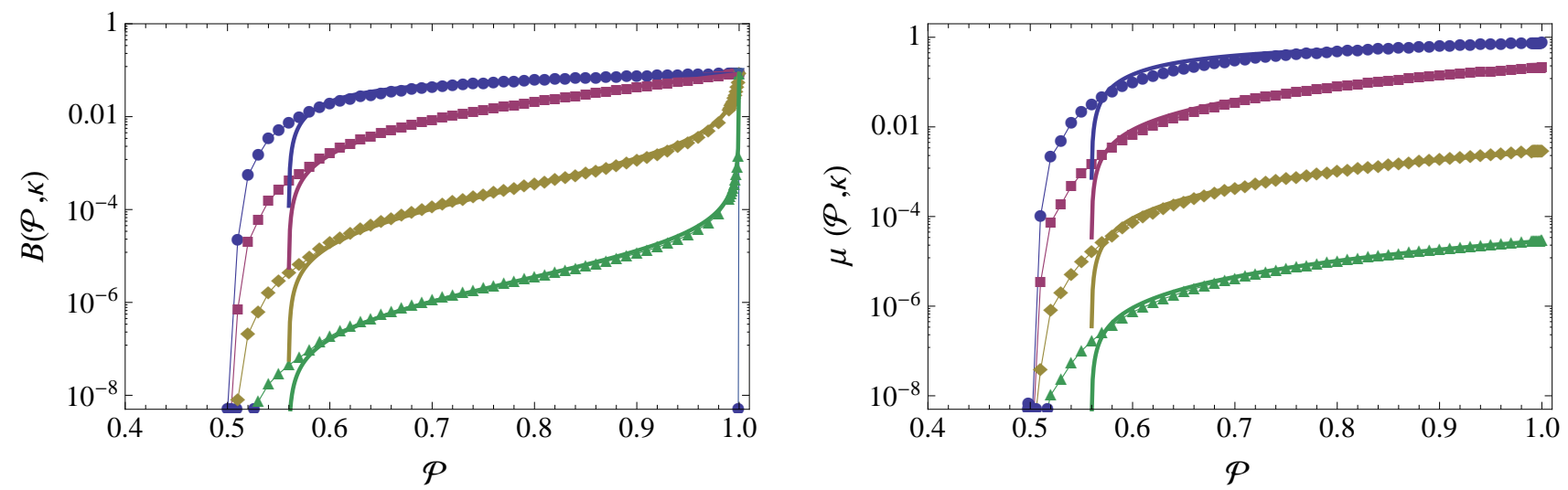

Figure 6. Bulk (on the left) and shear (on the right) moduli of the diluted diamond lattice as a function of $\mathcal{P}$, for $k=1$, and $\kappa=1$ (blue circles), $10^{-2}$ (red squares), $10^{-4}$ (yellow diamonds), and $10^{-6}$ (green triangles).

found in the EMT. We find $\mathcal{P}_{c} \approx 0.5$ in the simulations for the diamond lattice. We can use Eq. (11) and,

$z \mathcal{P}=\left(\sum_{i=0}^{z} i n_{i}\right) /\left(\sum_{i=0}^{z} n_{i}\right)$

along with a combinatorial calculation:

$n_{i}=N\left(\begin{array}{l}z \\ i\end{array}\right) \mathcal{P}^{i}(1-\mathcal{P})^{z-i}$

to show that at $\mathcal{P}=\mathcal{P}_{c}, r=r_{c} \approx 2.5$, which is close to the value $r_{c}=2.4$ that was obtained by $\mathrm{He}$ and Thorpe [14 using a different protocol.

The EMT equations provide analytic expressions for $\kappa_{m}$ and $k_{m}$ as a function of $\tilde{\kappa}$ and $\mathcal{P}$ in the vicinity of $\mathcal{P}=1$ and $\mathcal{P}=\mathcal{P}_{c}$, where

$\tilde{\kappa} \equiv \frac{\kappa}{k \ell_{0}^{2}}$,

is a unitless measure of the bending stiffness. We begin with $\mathcal{P}$ near one and seek expressions valid at small $\tilde{\kappa}$ where $\tilde{\kappa}_{m}$ is approximately equal to $\tilde{\kappa}$. Thus we expand $a^{*}$ and $b^{*}$ to linear order in $\tilde{\kappa}_{m}$. When $\tilde{\kappa}_{m}=0$ exactly, $a^{*}$ is ill defined because $\boldsymbol{D}_{s, \boldsymbol{q}}$ has a nullspace [dimension 1 (2) for the honeycomb (diamond) lattice)], and its inverse does not exist. When $\tilde{\kappa}_{m}$ is small, the projection of $\boldsymbol{D}_{\boldsymbol{q}}$ onto the null space of $\boldsymbol{D}_{s, \boldsymbol{q}}$ is proportional to $\tilde{\kappa}_{m}^{-1}$, but the contribution of this projection to the trace is zero because by definition $\boldsymbol{D}_{s, \boldsymbol{q}}$ is zero in its own nullspace. Thus, $a^{*}\left(\tilde{\kappa}_{m}\right)$ has a well-defined limit as $\tilde{\kappa}_{m} \rightarrow 0$ and a well-defined power series in $\tilde{\kappa}_{m}$. To linear order

$a^{*}=1-\alpha \tilde{\kappa}_{m}+\mathcal{O}\left(\tilde{\kappa}_{m}^{2}\right)$,

where $\alpha$ is 3.39 and 4.62 for the Honeycomb lattice and diamond lattices, respectively. A similar analysis can be applied to $b^{*}\left(\tilde{\kappa}_{m}\right)$, but with $\boldsymbol{D}_{b, \boldsymbol{q}}$ rather than $\boldsymbol{D}_{s, \boldsymbol{q}}$ having a nullspace. It is easier, however, to use the relation, Eq. (25) between $b^{*}$ and $a^{*}$ to obtain

$b^{*}=\frac{2 d-z}{z(z-1)}+\frac{\alpha}{z-1} \tilde{\kappa}_{m}+\mathcal{O}\left(\tilde{\kappa}_{m}^{2}\right)$. 
For small $\Delta \mathcal{P}=1-\mathcal{P}$ and $\tilde{\kappa}$, these results imply

$$
\begin{aligned}
\frac{k_{m}}{k} & =1-\frac{\Delta \mathcal{P}}{1-a^{*}} \\
& =1-\frac{\Delta \mathcal{P}}{\alpha \tilde{\kappa}_{m}}+\mathcal{O}\left(\Delta \mathcal{P} \tilde{\kappa}_{m}\right) \\
& =1-\frac{\Delta \mathcal{P} k_{m} \ell_{0}^{2}}{\alpha \kappa}+\mathcal{O}\left(\Delta \mathcal{P} \tilde{\kappa}_{m}, \Delta \mathcal{P}^{2}\right),
\end{aligned}
$$

where in the last line we have used Eqs. (13), (24) and (33). Thus,

$$
\frac{k_{m}}{k} \approx\left(1+\frac{1}{\alpha \tilde{\kappa}} \Delta \mathcal{P}\right)^{-1}
$$

and similarly,

$$
\frac{\kappa_{m}}{\kappa} \approx 1-\frac{2 z(z-1)}{z^{2}-2 d-z \alpha \tilde{\kappa}} \Delta \mathcal{P} \text {. }
$$

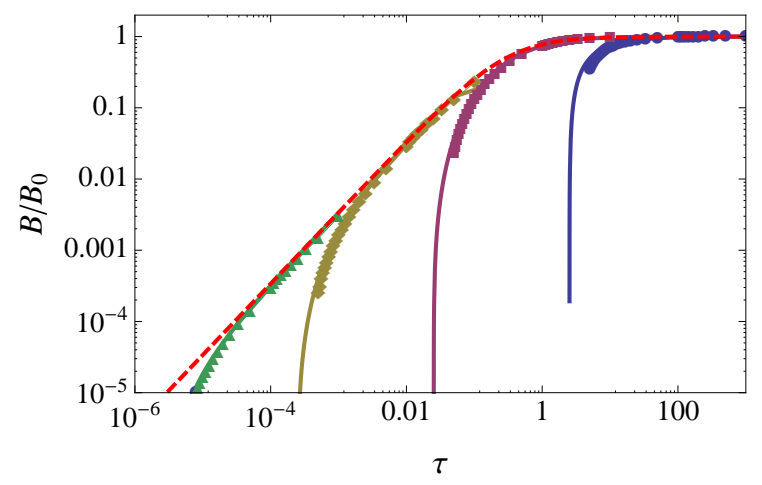

Figure 7. Scaling collapse for the bulk modulus of the honeycomb lattice near $\mathcal{P}=1$. The solid lines are the numerical solutions to the complete EMT equations, the dashed line the scaling solution of Eq. 40, and the data points are from simulations.

$\kappa_{m}$ is a function of $\Delta \mathcal{P}$ and $\kappa$ separately, but $k_{m}$ is a function of the ratio

$\tau=\frac{\tilde{\kappa}}{\Delta \mathcal{P}}$

only. We can then define a scaling function $\Xi$ for the bulk modulus $B$ near $\mathcal{P}=1$ :

$B=B_{0} \Xi(\tau)$,

where,

$\Xi(\tau)=\left(1+\frac{1}{\alpha \tau}\right)^{-1}$

and $B_{0}=A_{\mu} k$. When $\tau \rightarrow \infty,\left[\Delta \mathcal{P} \ll \kappa /\left(k \ell_{0}^{2}\right)\right]$, $B$ approaches the undiluted, $\kappa$-independent limit of $B_{0}=A_{B} k$ characterized by affine compression of all bonds. When $\tau \rightarrow 0\left[\Delta \mathcal{P} \gg \kappa /\left(k \ell_{0}^{2}\right)\right], B \rightarrow \tilde{\kappa} /(\alpha \Delta \mathcal{P})$, indicating that bonds in the diluted lattice are bent in response to isotropic compression and that response is nonaffine. Figure 7 plots $B / B_{0}$ as a function of $\tau$ for the honeycomb lattice obtained from full numerical solution of the EMT equations, the scaling function of Eq. (40), and numerical simulations. The three curves agree extremely well near $\mathcal{P}=1$. In addition, the simulation and full EMT curves follow each other closely, and both break away from the scaling curve at increasing values of $\Delta \mathcal{P}$ as $\tilde{\kappa}$ decreases. The shear modulus $\mu$ cannot be expressed as a scaling function of $\tau$. It approaches

$\mu_{0}=\left(A_{\mu} / \beta\right) k(1+(\gamma / \beta) \tilde{\kappa})^{-1}$

as $\Delta \rightarrow 0$.

To obtain analytic expressions for the bulk and shear moduli near the rigidity threshold at $\mathcal{P}=\mathcal{P}_{c}$ [given by Eq. (25)], we begin with the fact that $k_{m}=$ $\kappa_{m}=0$ at that point. Thus

$a^{*}\left(\kappa_{c}\right)=\mathcal{P}_{c}, \quad b^{*}\left(\kappa_{c}\right)=\mathcal{P}_{c}^{2}$,

where $\kappa_{c}(\approx 0.91$ for the honeycomb lattice and $\approx$ 0.30 for the diamond lattice) is the value of $\tilde{\kappa}_{m}$ at the rigidity threshold obtained by solving the $a^{*}\left(\kappa_{c}\right)$ equation for $\kappa_{c}$ with $\mathcal{P}_{c}$ given by Eq.(25). We are interested in what happens to lowest order in $\delta \mathcal{P}=$ $\mathcal{P}-\mathcal{P}_{c}$ as it increases from zero. To this end, we set $a^{*}=\mathcal{P}_{c}-c(\tilde{\kappa}) \delta \mathcal{P}$ and $b^{*}=\mathcal{P}_{c}^{2}(\tilde{\kappa})+c(\tilde{\kappa}) \delta \mathcal{P} /(z-1)$ with the coefficient $c(\tilde{\kappa})$ as yet undetermined. Then we use Eqs. (12) and (13) to obtain the ratio $\kappa_{m} /\left(\tilde{\kappa} k_{m} \ell_{0}^{2}\right)$ as a function of $\delta \mathcal{P}$. This ratio must approach $\kappa_{c} / \tilde{\kappa}$ in the limit $\delta \mathcal{P} \rightarrow 0$. But because of Eqs. (42), both the numerator and the denominator of this ratio are proportional to $\delta \mathcal{P}$ and the ratio itself depends on $c$ but not $\delta \mathcal{P}$ in the limit $\delta \mathcal{P} \rightarrow 0$. This limit equation determines $c(\tilde{\kappa})$ :

$c(\tilde{\kappa})=\frac{\kappa_{c}-2(z-1) \mathcal{P}_{c} s_{c} \tilde{\kappa}}{\kappa_{c}+\tilde{\kappa} s_{c}}$

where

$s_{c}=\frac{1-\mathcal{P}_{c}}{(z-1)\left(1-\mathcal{P}_{c}^{2}\right)}$

The equations for $k_{m}$ and $\kappa_{m}$ then become

$\frac{k_{m}}{k} \approx \frac{1-c(\tilde{\kappa})}{1-\mathcal{P}_{c}} \delta \mathcal{P}$,

and

$\frac{\kappa_{m}}{\kappa} \approx \frac{2(z-1) \mathcal{P}_{c}+c(\tilde{\kappa})}{(z-1)\left(1-\mathcal{P}_{c}^{2}\right)} \delta \mathcal{P}$.

Equations (45) and (46) predict linear behavior of the elastic moduli near $\mathcal{P}=\mathcal{P}_{c}$. However, scaling collapse plots of the simulation results suggest that $B$ and $\mu$ scale with $\delta \mathcal{P}^{1.6}$, for both honeycomb and diamond lattices, as it is shown in Figure 8 for the shear modulus. The exponent 1.6, which is near the value 1.5 found by $\mathrm{He}$ and Thorpe [14, is obtained as the best fit of our data near $\delta \mathcal{P}=0$. However, 

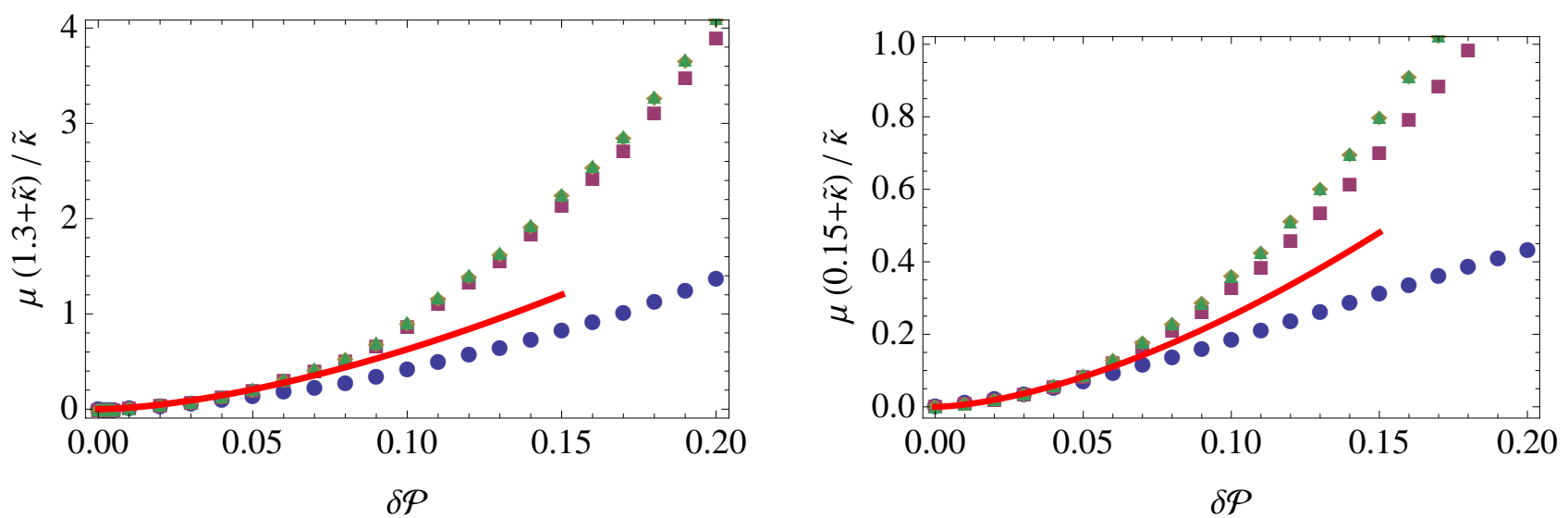

Figure 8. Scaling collapse for the shear modulus for the honeycomb (left) and diamond (right) lattices near $\mathcal{P}=\mathcal{P}_{c}$, for $k=1$, and $\kappa=1$ (blue circles), $10^{-2}$ (red squares), $10^{-4}$ (yellow diamonds), and $10^{-6}$ (green triangles). The red line is a best fit near $\delta \mathcal{P}=0$. The smaller values at $\tilde{\kappa}=1$ are consistent with the EMT expression for $\mu$ (Eq. (28). The simulations power-law decay is characterized by an approximate exponent of 1.6 for both lattices.

we note that a log-log plot of this data is very noisy near the rigidity percolation threshold, and that a more precise determination of the exponent requires a careful analysis of finite-size scaling, which is beyond the goal of this manuscript. Finally, the simulation nonaffinity ratio for the bulk modulus $\Gamma_{B}$, as a function of $\mathcal{P}$, is shown in Fig. 9 .

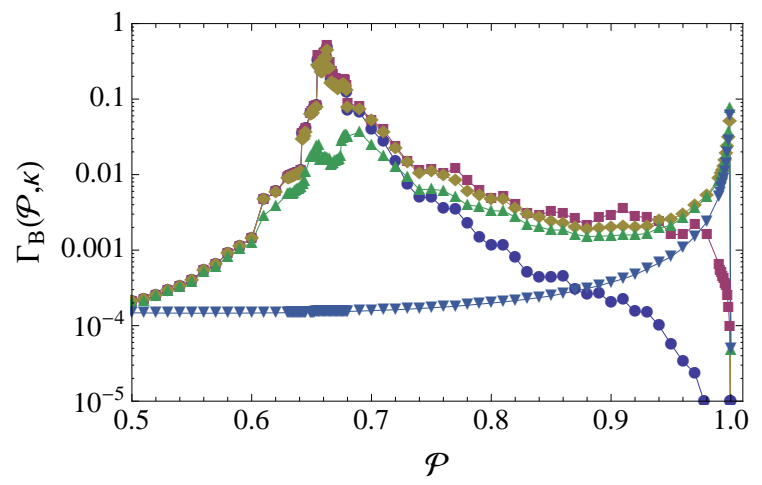

Figure 9. Nonaffinity ratio of the bulk modulus $\Gamma_{B}$ for the honeycomb lattice as a function of the probability $\mathcal{P}$.

In our effective medium theory, the ratio of $\mu$ to $B$ is a function of $\tilde{\kappa}_{m}$ :

$\frac{\mu}{B}=\frac{A_{\mu}}{A_{B}}\left(\frac{\beta}{\tilde{\kappa}_{m}}+\gamma\right)^{-1}$.

At the rigidity threshold at which $\tilde{\kappa}_{m}=\kappa_{c}$, this ratio becomes

$\frac{\mu}{B}= \begin{cases}1.608 & \text { Honeycomb } \\ 6.097 & \text { Diamond }\end{cases}$
The Poisson ratio at threshold of the honeycomb lattice,

$\sigma_{P}=\frac{1-(\mu / B)}{1+(\mu / B)}=-0.233$,

is thus negative. The Poisson ratio of the diamond lattice, which continues to have macroscopic cubic symmetry in the EMT depends on direction of stresses, and we do not calculate it.

\section{Summary}

To summarize, we have studied phonon and elastic properties of the honeycomb and diamond lattices with nearest-neighbor interactions in the harmonic approximation and rotationally invariant bending interactions. These lattices are under-coordinated, in the sense that their average coordination numbers are smaller than the isostatic limit $z_{c}=2 d$. They present oscillation modes which reduce to floppy modes over the entire Brillouin zone when the bending energy coupling constant vanishes. We implement disorder by assigning a probability distribution to the existence of each bond in the lattices. When the number of diluted bonds hits a critical value, there is a rigidity percolation phase transition at which both bulk and shear moduli vanish. We employ numerical simulations and an effective-medium theory to study scaling behavior near $\mathcal{P}=1$, where the effectivemedium theory reproduces the exact results for the undiluted lattice, and near the critical probability $\mathcal{P}=\mathcal{P}_{c}$. The scaling behavior predicted by the EMT near $\mathcal{P}=1$ is fairly well satisfied by the numerical simulations. However, the EMT predicts a different decay near $\mathcal{P}=\mathcal{P}_{c}$, with the elastic moduli 
proportional to $\delta \mathcal{P}$, in contrast with the simulation results, where $B, \mu \sim \delta \mathcal{P}^{1.6}$.

\section{Acknowledgement}

This work was supported by the Brazilian agencies Fapesp and Capes (DBL), and the NSF under Grants No. DMR-1104707 (TCL) and No. DMR-1120901 (OS). DBL thanks James Sethna for useful comments and suggestions.

\section{Appendix A. Derivation of the bending energy in terms of displacements}

This appendix will show that bending potentials can be cast as a manifestly rotationally invariant combination of displacement vectors that obey the Keating Rules [23. First some notation: Let $\mathbf{r}_{\mu}$ be the equilibrium reference position of site $\mu$, and let $\mathbf{R}_{\mu}=\mathbf{r}_{\mu}+\mathbf{u}_{\mu}$ be the position of site $\mu$ after distortion, where $\mathbf{u}_{\mu}$ is the displacement vector. Now, consider two bonds, which we label $a$ and $b$, sharing a common site, which we label as site 0 . Bond $a$ connects site 1 to site 0 and bond $b$ connects site 2 to site 0 . Then define

$\mathbf{r}_{a}=\mathbf{r}_{1}-\mathbf{r}_{0} ; \quad \mathbf{r}_{b}=\mathbf{r}_{2}-\mathbf{r}_{0}$

$\mathbf{R}_{a}=\mathbf{R}_{0}-\mathbf{R}_{1} \equiv \mathbf{r}_{a}+\mathbf{u}_{a} ; \quad \mathbf{u}_{a}=\mathbf{u}_{1}-\mathbf{u}_{0}$

$\mathbf{R}_{b}=\mathbf{R}_{2}-\mathbf{R}_{1} \equiv \mathbf{r}_{b}+\mathbf{u}_{b} ; \quad \mathbf{u}_{b}=\mathbf{u}_{2}-\mathbf{u}_{0}$.

$\mathbf{r}_{a, b}$ is the equilibrium vector and $\mathbf{R}_{a, b}$ the stretched vector for bond $a(b)$.

We seek an energy that depends on the angle $\beta_{a b}$ between bonds $a$ and $b$. To define the $\beta_{a b}$, we assume that it lies between 0 and $\pi$ so that its sine is positive (extension to negative $\beta$ is possible but not relevant to our current interest). Then

$\sin \beta_{a b}=\frac{\left|\mathbf{R}_{a} \times \mathbf{R}_{b}\right|}{R_{a} R_{b}} ; \quad \sin \beta_{0}=\frac{\mathbf{r}_{a} \times \mathbf{r}_{b}}{r_{a} r_{b}}$,

where $R_{a}=\left|\mathbf{R}_{a}\right|$ and $r_{a}=\left|\mathbf{r}_{a}\right|$, and we set the bending energy of the bond-pair $a b$ to

$$
\begin{aligned}
E_{b} & =\frac{1}{2} \tilde{\kappa}\left(\beta_{a b}-\beta_{0}\right)^{2}, \quad \text { or } \\
& =\frac{1}{2} \tilde{\kappa}\left(\sin ^{-1} \frac{\left|\mathbf{R}_{a} \times \mathbf{R}_{b}\right|}{R_{a} R_{b}}-\sin ^{-1} \frac{\left|\mathbf{r}_{a} \times \mathbf{r}_{b}\right|}{r_{a} r_{b}}\right)^{2} .
\end{aligned}
$$

It is clear that this energy is rotationally invariant.

We can now express these energies in terms of

$$
\begin{aligned}
v_{a} & =\frac{1}{2}\left(R_{a}^{2}-r_{a}^{2}\right)=\frac{1}{2}\left(2 \mathbf{r}_{a} \cdot \mathbf{u}_{a}+\mathbf{u}_{a} \cdot \mathbf{u}_{a}\right) \rightarrow r_{a i} r_{a j} u_{i j} \\
v_{b} & =\frac{1}{2}\left(R_{b}^{2}-r_{b}^{2}\right)=\frac{1}{2}\left(2 \mathbf{r}_{b} \cdot \mathbf{u}_{b}+\mathbf{u}_{b} \cdot \mathbf{u}_{b}\right) \rightarrow r_{b i} r_{b j} u_{i j} \\
v_{a b} & =\frac{1}{2}\left(\mathbf{R}_{a} \cdot \mathbf{R}_{b}-\mathbf{r}_{a} \cdot \mathbf{r}_{b}\right) \\
& =\frac{1}{2}\left(\mathbf{r}_{a} \cdot \mathbf{u}_{b}+\mathbf{r}_{b} \cdot \mathbf{u}_{a} \mathbf{u}_{a} \cdot \mathbf{u}_{b}\right) \rightarrow r_{a i} r_{b j} u_{i j},
\end{aligned}
$$

where $i$ and $j$ are Cartesian indices $x, y, z$. The final forms are the long-wavelength continuum limits with

$u_{i j}=\frac{1}{2}\left(\partial_{i} u_{j}+\partial_{j} u_{i}+\partial_{i} \mathbf{u} \cdot \partial_{j} \mathbf{u}\right)$

the usual rotationally invariant nonlinear Lagrangian strain tensor and $\mathbf{u}(\mathbf{x})$ the displacement field at spacepoint $\mathbf{x}$. These limits were obtained using $\mathbf{r}_{0} \equiv$ $\mathbf{x}$ as the reference point and $\mathbf{u}_{a} \rightarrow r_{a i} \partial_{i} \mathbf{u}(\mathbf{x})$ and $\mathbf{u}_{b} \rightarrow r_{b i} \partial_{i} \mathbf{u}(\mathbf{x}) . \quad v_{a}$ and $v_{b}$ are the forms that normally appear in central-force models and $v_{a b}$ is the quantity that Keaton introduces. The continuum limits guarantee that a continuum elastic energy constructed from the bending energy of Eq. A.4 will be a function of $u_{i j}$ only, as it must be to be rotationally invariant. It should be noted, however, that the complete bending energies have terms higher order in derivatives.

We express the quantities in Eq. A.4 in terms of $v_{a}, v_{b}$ and $v_{a b}$ :

$\left|\mathbf{R}_{a} \times \mathbf{R}_{b}\right|^{2}=R_{a}^{2} R_{b}^{2}-\left(\mathbf{R}_{a} \cdot \mathbf{R}_{b}\right)^{2}=\left|\mathbf{r}_{a} \times \mathbf{r}_{b}\right|^{2}+2 V_{a b}$
$V_{a b}=\left(r_{a}^{2} v_{b}+r_{b}^{2} v_{a}-2 \mathbf{r}_{a} \cdot \mathbf{r}_{b} v_{a b}\right)+2\left(v_{a} v_{b}-v_{a b}^{2}\right)$

The form of this expression depends on whether $\mathbf{r}_{a}$ and $\mathbf{r}_{b}$ are parallel or not: if they are parallel, $\mathbf{r}_{a} \times \mathbf{r}_{b}=0$ and $\beta_{0}=0, \pi$. This limit applies to models for filamentous lattices [8, 20, 22]. The other limit $\beta_{0} \neq 0$ applies to the honeycomb and diamond lattices. It is important to note that Eq. (A.6) and thus the bending energy $E_{b}$ depends on both the Keating part $v_{a b}$ and the "central-force" parts $v_{a}$ and $v_{b}$, but by construction it depends only on the the rotation angle between bonds and not on any stretch in the bonds. The Keating energy depends on stretching as well as bending and contributes to both the bulk and shear moduli. The bulk modulus does not depend on a pure bending energy of the type we discuss. There is no change in the angle between bonds under uniform compression. With the above definitions,

$$
\frac{\left|\mathbf{R}_{a} \times \mathbf{R}_{b}\right|}{R_{a} R_{b} \sin \beta_{0}}=\left(\frac{1+2 V_{a b} /\left|\mathbf{r}_{a} \times \mathbf{r}_{b}\right|^{2}}{\left(1+2 v_{a} / r_{a}^{2}\right)\left(1+2 v_{b} / r_{b}^{2}\right)}\right)^{1 / 2}
$$

and

$\beta-\beta_{0} \approx \tan \beta_{0}\left(\frac{V_{a b}}{\left|\mathbf{r}_{a} \times \mathbf{r}_{b}\right|^{2}}-\frac{v_{a}}{r_{a}^{2}}-\frac{v_{b}}{r_{b}^{2}}\right)$

in the small-displacement limit.

Equations Eq. A.4 and (A.6) provide a complete expression for bending energies in terms of nonlinear functions of the nonlinear "discrete" strain functions $v_{a}, v_{b}$, and $v_{a b}$. We are often interested in the harmonic limit of these functions. We begin with the case $\beta_{0}>0$, and we expand to lowest order in $\mathbf{u}_{a}$ and $\mathbf{u}_{b}$

$v_{a} \rightarrow \mathbf{r}_{a} \cdot \mathbf{u}_{a}, \quad v_{a b} \rightarrow \frac{1}{2}\left(\mathbf{r}_{a} \cdot \mathbf{u}_{b}+\mathbf{r}_{b} \cdot \mathbf{u}_{a}\right)$, 
and

$$
\begin{aligned}
V_{a b} & \rightarrow r_{a}^{2} \mathbf{r}_{b} \cdot \mathbf{u}_{b}+r_{b}^{2} \mathbf{r}_{a} \cdot \mathbf{u}_{a}-\mathbf{r}_{a} \cdot \mathbf{r}_{b}\left(\mathbf{r}_{a} \cdot \mathbf{u}_{b}+\mathbf{r}_{b} \cdot \mathbf{u}_{a}\right) \\
& =r_{b}^{2} \mathbf{r}_{a} \cdot \mathbf{u}_{a}^{\perp b}+r_{a}^{2} \mathbf{r}_{b} \cdot \mathbf{u}_{b}^{\perp a},
\end{aligned}
$$

where $\mathbf{u}_{a}^{\perp b}$ is the projection of $\mathbf{u}_{a}$ onto the space perpendicular to $\mathbf{r}_{b}$, i.e. $u_{a i}^{\perp b}=P_{i j}^{b} u_{a j}$, where $P_{i j}^{b}=$ $\delta_{i j}-\hat{r}_{b i} \hat{r}_{b j}$, with $\hat{\mathbf{r}}_{b}=\mathbf{r}_{b} / r_{b}$, is the projection operator onto the plane perpendicular to $\mathbf{r}_{b}$. Then with the aid Eqs. A.9 and A.10 and the relation

$$
\begin{gathered}
\left(\frac{1}{\sin ^{2} \beta_{0}}-1\right) \hat{r}_{a i} P_{i j}^{b} u_{a j}-\hat{r}_{a j} u_{a j} \\
=\frac{\cos \beta_{0}}{\sin ^{2} \beta_{0}} \hat{r}_{b i} P_{i j}^{a} u_{a j},
\end{gathered}
$$

the energy $E_{b}$ to harmonic order is

$E_{b}^{\mathrm{har}}=\frac{\tilde{\kappa}}{2 \sin ^{2} \beta_{0}}\left(\hat{\mathbf{r}}_{b} \cdot \frac{\mathbf{u}_{a}^{\perp a}}{r_{a}}+\hat{\mathbf{r}}_{a} \cdot \frac{\mathbf{u}_{b}^{\perp b}}{r_{b}}\right)^{2}$

Note that this energy depends only on displacements perpendicular to equilibrium bond directions and thus it does not induce any bond compression. Setting $\left.\tilde{\kappa}=\kappa \sin \beta_{0}\right), a=l k, b=l m$, and $r_{a}=r_{b}=l_{0}$ leads to Eq. (41). If only the $v_{a b}$ part were kept, $E_{b}^{\text {har }}$ reduces to the form used in reference 14 .

When $\mathbf{r}_{a}=r_{a} \mathbf{e}$ and $\mathbf{r}_{b}=-r_{b} \mathbf{e}$ are anti-parallel, $\beta_{0}=\pi, \sin ^{2} \beta=2 V_{a b} / R_{a}^{2} R_{b}^{2}$, and the linear part of $V_{a b}$ vanishes:

$$
\begin{aligned}
V_{a b}^{(1)}=r_{b}^{2} r_{a} \mathbf{e} \cdot \mathbf{u}_{a}-r_{a}^{2} r_{b} \mathbf{e} \cdot \mathbf{u}_{b} \\
\quad+r_{a} r_{b}\left(r_{a} \mathbf{e} \cdot \mathbf{u}_{b}-r_{b} \mathbf{e} \cdot \mathbf{u}_{a}\right)=0 .
\end{aligned}
$$

The quadratic part of $V_{a b}$ is

$V_{a b}^{(2)}=\frac{1}{2}\left(r_{a} \mathbf{u}_{b}^{\perp}+r_{b} \mathbf{u}_{a}^{\perp}\right)^{2}$

When $\mathbf{u}_{a i}^{\perp}=\left(\delta_{i j}-e_{i} e_{j}\right) u_{a, j}$, and $E_{b}$ becomes $\tilde{\kappa} V_{a b}^{(2)} /\left(r_{a}^{2} r_{b}^{2}\right)$ to harmonic order. If we had chosen $\mathbf{r}_{b}=\mathbf{r}_{0}-\mathbf{r}_{2}$ rather than $\mathbf{r}_{b}=\mathbf{r}_{2}-\mathbf{r}_{0}$, then $\beta_{0}=0$, and there would be a minus sign in Eq. A.14.

\section{References}

[1] M. Thorpe. J. Non-Cryst. Solids, 57:355, 1983.

[2] S. Feng and P. N. Sen. Phys. Rev. Lett., 52:216, 1984.

[3] S. Alexander. Phys. Rep., 296:65, 1998.

[4] M. Wyart. Ann. Phys. (Paris), 30:1, 2005.

[5] S. F. Edwards and D. V. Grinev. Phys. Rev. Lett., 82:5397, 1999.

[6] A. V. Tkachenko and T. A. Witten. Phys. Rev. E, 60:687, 1999 .

[7] K. D. Hammonds, M. T. Dove, A. P. Giddy, V. Heine, and B. Winkler. Am. Mineral., 81:1057, 1996.

[8] C. P. Broedersz and F. C. MacKintosh. Modeling semiflexible polymer networks. Rev. Mod. Phys. 86, 995, $86,2014$.

[9] C. P. Broedersz, X. Mao, T. C. Lubensky, and F. C. MacKintosh. Criticality and isostaticity in fibre networks. Nat. Phys., 7:983-988, 2011.

[10] M. Das, D. A. Quint, and J. M. Schwarz. PLoS One, 7:e35939, 2012.
[11] Martin Lenz. Geometrical origins of contractility in disordered actomyosin networks. Physical Review $X$, 4(4):041002, 2014.

[12] Pierre Ronceray and Martin Lenz. Connecting local active forces to macroscopic stress in elastic media. Soft Matter, 11(8):1597-1605, 2015.

[13] S. Feng, M. F. Thorpe, and E. Garboczi. Phys. Rev. B, $31: 276,1985$

[14] H. He and M. F. Thorpe. Phys. Rev. Lett., 54:2107, 1985.

[15] X. Mao and T. C. Lubensky. Phys. Rev. E, 83:011111, 2011.

[16] X. Mao, O. Stenull, and T. C. Lubensky. Phys. Rev. E, 87:042601, 2013

[17] X. Mao, O. Stenull, and T. C. Lubensky. Phys. Rev. E, 87:042602, 2013

[18] J. C. Maxwell. Philos. Mag., 27:294, 1865.

[19] A. Souslov, A. J. Liu, and T. C. Lubensky. Phys. Rev. Lett., 103:205503, 2009.

[20] David A. Head, Alex J. Levine, and F. C. MacKintosh. Deformation of cross-linked semiflexible polymer networks. Phys. Rev. Lett., 91(10):108102, 2003.

[21] D. A. Head, A. J. Levine, and F. C. MacKintosh. Distinct regimes of elastic response and deformation modes of cross-linked cytoskeletal and semiflexible polymer networks. Phys. Rev. E, 68(6):061907, 2003.

[22] Jan Wilhelm and Erwin Frey. Elasticity of stiff polymer networks. Phys. Rev. Lett., 91(10):108103, 2003.

[23] P. N. Keating. Phys. Rev., 145:637, 1966.

[24] N. W. Ashcroft and N. D. Mermin. Solid state physics. Hold, Rinehart, and Winston, New York, 1976.

[25] M. Das, F. C. MacKintosh, and A. J. Levine. Phys. Rev. Lett., 99:038101, 2007.

[26] F. Yonezawa and T. Odagaki. Analytic extension of the coherent potential approximation to clusters. Solid State Communications, 27(11):1199 - 1202, 1978.

[27] J. C. Phillips. J. Non-Cryst. Solids, 34:153, 1979

[28] J. C. Phillips. J. Non-Cryst. Solids, 43:37, 1981. 